\title{
Attack Resistance of Power-Law Random Graphs in the Finite- Mean, Infinite-Variance Region
}

\author{
Ilkka Norros and Hannu Reittu
}

Abstract. We consider a conditionally Poisson random-graph model in which the mean degrees, "capacities," follow a power-tail distribution with finite mean and infinite variance. Such a graph of size $N$ has a giant component that is supersmall in the sense that the typical distance between vertices is of order $\log \log N$. The shortest paths travel through a core consisting of nodes with high mean degrees. In this paper we derive upper bounds for the distance between two random vertices when an upper part of the core is removed, including the case that the whole core is removed.

\section{Introduction}

Power-law (or scale-free) random graphs have become popular objects of both applied and theoretical interest, because they are simple to define and generate and yet share some important characteristics with many large and complex realworld networks. The mathematical models help us to understand how and under what conditions those characteristics emerge, and this understanding can be valuable also in the design of new artificial networks.

The general characteristic of power-law random graphs is that their degree distribution possesses a regularly varying tail, the most interesting region of tail exponents being that of finite mean and infinite variance. Several models with these features have been studied. In this paper, we work with the conditionally Poisson random graph [Norros and Reittu 06], a modification of the expected degree sequence model of [Chung and $\mathrm{Lu}$ 03]. We draw an i.i.d. sequence of

(C) A K Peters, Ltd.

I542-7951/08 \$0.50 per page 
mean degrees, "capacities," that follow a Pareto distribution with finite mean and infinite variance.

Our graph of size $N$ has a giant component that is ultrasmall in the sense that the typical distance between vertices is proportional to $\log \log N$ (more precisely, this is an upper bound, like most of our distance results), whereas a similar random graph whose degrees have the same mean but finite variance cannot offer better than $\log N$ scalability of distances. Remarkably, the speciality of the infinite variance case is the emergence of a "core network" consisting of nodes of high degree, through which the short paths typically travel. It is now natural to ask what happens to these distances if vertices with highest degrees are deleted. Our main findings can be summarized as follows:

(i) Any deletion of core vertices leaves the asymptotic size of the largest giant component intact.

(ii) If vertices with capacity greater than $N^{\gamma}$ are deleted, $\gamma$ not depending on $N$, then paths that would have gone through the deleted vertices can be repaired using "backup paths" that increase the distances only by a constant depending on $\gamma$ but not on $N$. This increase is negligible on the $\log \log N$ scaling of distances.

(iii) If the whole core is removed (for the exact meaning of this, see Section 2), the distances still scale slightly better than $\log N$.

Because of structural similarity (compare [Reittu and Norros 04] with [Norros and Reittu 06]), we conjecture that our results are transferable to the configuration model of [Newman et al. 01, Reittu and Norros 04]. Perhaps they can be extended even to preferential attachment models in the finite-mean and infinitevariance region, since van der Hofstad and Hooghiemstra showed recently that these models share with the previously mentioned models the $\log \log$ scalability of distances [van der Hofstad and Hooghiemstra 07].

It is worth pointing out how our "invulnerability" results are related to the well-known vulnerability result of [Bollobás and Riordan 03] (which gives rigor to an earlier observation in [Albert et al. 00]). The models are different, of course. As regards the results, however, the main difference is that the attacks considered in [Bollobás and Riordan 03] delete a positive fraction of vertices, choosing the largest ones in terms of degree, whereas our core consists of an asymptotically negligible fraction of vertices. It is plausible that such a more massive attack would have consequences in our model similar to those found in [Bollobás and Riordan 03].

The next section specifies the graph model and reviews the relevant results of [Norros and Reittu 06]. Some of them are slightly sharpened in Section 3. 
Section 4 applies results on the diameter of classical random graphs to measure the core network in the horizontal direction. The main result on robustness against core losses is stated and proven in Section 5 .

\section{Model and Earlier Results}

Throughout this paper, we work with the following conditionally Poisson powerlaw random graph model [Norros and Reittu 06]. There are $N$ vertices that possess, respectively, i.i.d. "capacities" $\Lambda_{1}, \ldots, \Lambda_{N}$ with distribution

$$
\mathbb{P}(\Lambda>x)=x^{-\tau+1}, \quad x \in[1, \infty), \quad \tau \in(2,3) .
$$

Given $\boldsymbol{\Lambda}=\left(\Lambda_{1}, \ldots, \Lambda_{N}\right)$, each pair of vertices $\{i, j\}$ is connected with $E_{i j}$ edges, where

$$
E_{i j} \sim \operatorname{Poisson}\left(\frac{\Lambda_{i} \Lambda_{j}}{L_{N}}\right), \quad L_{N}=\sum_{j=1}^{N} \Lambda_{j},
$$

and the $E_{i j}$ 's are independent. Loops, i.e., the case $i=j$, are included for principle, although they have no significance in the present study. Given $\boldsymbol{\Lambda}$, the degree of vertex $i$ then has the distribution Poisson $\left(\Lambda_{i}\right)$. The resulting random graph is denoted by $G_{N}$.

The following coupling between neighborhood shells and branching processes will play an important role, as it did in [Norros and Reittu 06]. Fix $N$, and assume that the sequence $\boldsymbol{\Lambda}$ has been generated and probabilities are now conditioned on it. We shall consider a marked branching process, i.e., a branching process in which each individual is associated with some element of the mark space $\{1, \ldots, N\}$. More specifically, let us define a process $(Z, \mathbf{J})=\left(Z_{n},\left(J_{n, i}\right)\right)$, where $Z_{n}$ is the size of generation $n$, and $J_{n, i} \in\{1, \ldots, N\}$ is the mark of member $i$ of generation $n$. We set $Z_{0} \equiv 1$ and take $J_{0,1}$ from the uniform distribution $U\{1, \ldots, N\}$. The process then proceeds so that an individual bearing mark $i$ gives birth independently to a Poisson $\left(\Lambda_{i} \Lambda_{j} / L_{N}\right)$ distributed number of $j$-marked members of the next generation for each $j=1, \ldots, N$.

On the other hand, we consider the neighborhood sequence around a random vertex of $G_{N}$. Take a vertex $i_{0}$ from uniform distribution, and define recursively

$$
\begin{aligned}
\mathcal{N}_{0}\left(i_{0}\right) & =\left\{i_{0}\right\}, \\
\mathcal{N}_{k+1}\left(i_{0}\right) & =\left\{j \in\left(\bigcup_{l=0}^{k} \mathcal{N}_{l}\left(i_{0}\right)\right)^{c}: j \leftrightarrow \mathcal{N}_{k}\left(i_{0}\right)\right\} .
\end{aligned}
$$

The following coupling was proven in [Norros and Reittu 06]. 
Proposition 2.I. Let $(Z, \mathbf{J})$ be the marked branching process defined above. Define a reduced process by proceeding generation by generation, i.e., in the order

$$
J_{0,1} ; \quad J_{1,1}, J_{1,2}, \ldots, J_{1, Z_{1}} ; J_{2,1}, \ldots
$$

and pruning (that is, deleting) from $(Z, \mathbf{J})$ each individual whose mark has previously appeared, together with all its descendants (these are considered as not seen in the procedure). Denote the resulting finite process by $(\hat{Z}, \hat{\mathbf{J}})$, and let $\hat{\mathcal{J}}_{k}$ be the set of the marks in generation $k$ of the reduced process. Then the sequence of the sets $\hat{\mathcal{J}}_{k}$ has the same distribution as the sequence $\mathcal{N}_{k}$.

Moreover, we observed that the size of each generation and its marks can be generated independently. Denote by $q_{N}$ the distribution

$$
q_{N}(j) \doteq \frac{\Lambda_{j}}{L_{N}}, \quad j=1, \ldots, N,
$$

and by $\pi^{*}$ the mixed Poisson distribution Poisson $(\Gamma)$, where $\Gamma$ is a random variable with distribution

$$
\mathbb{P}(\Gamma \in \mathrm{d} x)=x \mathbb{P}(\Lambda \in \mathrm{d} x) / \mathbb{E}\{\Lambda\} .
$$

Obviously, the distribution of Poisson $\left(\Lambda_{J^{(N)}}\right), J^{(N)} \sim q_{N}$, converges weakly to $\pi^{*}$ in probability.

Let $J_{1}^{(N)}, J_{2}^{(N)}, \ldots$ be i.i.d. random variables with distribution $q_{N}$, and let $J_{0}^{(N)}$ be a random variable with uniform distribution on $\{1, \ldots, N\}$, independent of the previous ones; we often suppress the superscript $(N)$. Start now with $\left(\tilde{Z}_{0}=1, J_{0}\right)$, and proceed generationwise by drawing the size of the $(n+1)$ st generation from the distribution

$$
\tilde{Z}_{n+1} \sim \text { Poisson }\left(\sum_{i=M_{n-1}+1}^{M_{n}} \Lambda_{J_{i}}\right),
$$

where $M_{-1}=0, M_{n}=\sum_{0}^{n} \tilde{Z}_{k}$, and then giving each individual a fresh mark from the sequence $J_{M_{n}+1}, J_{M_{n}+2}, \ldots$.

Proposition 2.2. The marked generation sequence $\left(Z_{n},\left(J_{n, i}\right)\right)$ is stochastically equivalent to

$$
\left(\tilde{Z}_{n},\left(J_{M_{n-1}+1}, \ldots, J_{M_{n}}\right)\right) .
$$

We next turn to the definition of the core network mentioned in the introduction. Fix an increasing function $\ell: \mathbb{N} \rightarrow \mathbb{R}$ such that

$$
\ell(1)=1, \quad \frac{\ell(N)}{\log \log \log N} \rightarrow 0, \quad \frac{\ell(N)}{\log \log \log \log N} \rightarrow \infty \quad \text { as } N \rightarrow \infty,
$$


and define

$$
\epsilon(N):=\frac{\ell(N)}{\log N}
$$

Note that

$$
\epsilon(N) \rightarrow 0, \quad N^{\epsilon(N)}=e^{\ell(N)} \rightarrow \infty \quad \text { as } N \rightarrow \infty
$$

Define recursively the functions

$$
\begin{aligned}
& \beta_{0}(N)=\frac{1}{\tau-1}+\frac{\epsilon(N)}{\tau-2}, \\
& \beta_{j}(N)=(\tau-2) \beta_{j-1}(N)+\epsilon(N), \quad j=1,2, \ldots,
\end{aligned}
$$

and set

$$
k^{*} \doteq\left\lceil\frac{\log \log N}{-\log (\tau-2)}\right\rceil \text {. }
$$

For $N$ sufficiently large, we have $\beta_{0}(N)>\epsilon(N) /(3-\tau)$, and the sequence $\left(\beta_{k}(N)\right)_{k=0,1, \ldots}$ decreases toward the limit value $\epsilon(N) /(3-\tau)$. With $k=k^{*}$, we are already in the $\epsilon(N)$ order of magnitude:

$$
\beta_{k^{*}(N)}(N) \leq \epsilon(N)\left(1+\sum_{i=0}^{\infty}(\tau-2)^{i}\right)=\frac{4-\tau}{3-\tau} \epsilon(N)
$$

The key of our analysis of $G_{N}$ is the notion of its core $C$, consisting of all vertices with capacity greater than $N^{\beta_{k} *}$. Note that the exact boundary of the core depends on $\ell(N)$ and is thus somewhat arbitrary.

By the definition of $\epsilon(N)$, we can choose a natural number $\kappa=\kappa(N)$ such that

$$
\frac{\kappa(N)}{N^{\theta \epsilon(N)}} \rightarrow \infty, \quad \frac{\kappa(N)}{k^{*}(N)} \rightarrow 0, \quad \text { as } N \rightarrow \infty,
$$

where $\theta=(\tau-2)(4-\tau) /(3-\tau)$. We can now collect the main results of [Norros and Reittu 06] in the following theorem:

\section{Theorem 2.3.}

1. The graph $G_{N}$ has a.a.s. (asymptotically almost surely) a giant component whose relative size approaches the value

$$
1-\sum_{j=1}^{\infty} \mathbb{P}(D=j) \mathbb{P}\left(Z_{\infty}^{\left(\pi^{*}\right)}=0\right)^{j}
$$

where $D$ is distributed as the conditionally Poisson variable Poisson $(\Lambda)$, and $\left(Z_{n}^{\left(\pi^{*}\right)}\right)$ is a Galton-Watson branching process with offspring distribution $\pi^{*}$. 
2. A random vertex of the giant component is a.a.s. connected with the core $C$ in fewer than $\kappa(N)$ hops. The distribution of the capacity of the first core vertex found in a random-order breadth-first neighborhood search is asymptotically identical to the distribution of $J_{n_{C}}^{(N)}$, where $n_{C}=\inf \left\{n: J_{n}^{(N)} \in C\right\}$.

3. A random vertex of the core is a.a.s. connected with the vertex $i^{*}$ with highest capacity in at most $k^{*}$ hops.

4. As a consequence of the previous items, the distance between two randomly chosen vertices of the giant component is at most $2 k^{*}(N)(1+o(1))$ a.a.s.

Set $\mathcal{T}_{\gamma, \delta}=\left\{i \in\{1, \ldots, N\}: \Lambda_{i} \in\left(N^{\gamma}, N^{\delta}\right]\right\}$. The idea of the proof of claim 3 of Theorem 2.3 is that if the core is divided into "tiers"

$$
\begin{aligned}
& V_{0}=\left\{i^{*}\right\}, \\
& V_{1}=\mathcal{T}_{\beta_{1}, \infty} \backslash\left\{i^{*}\right\}, \\
& V_{k}=\mathcal{T}_{\beta_{k}, \beta_{k-1}}, \quad k=2, \ldots, k^{*},
\end{aligned}
$$

then for any $k>0$, a random vertex of tier $k$ has a.a.s. an edge to $V_{k-1} \cup \cdots \cup V_{0}$.

\section{Auxiliary Results}

In this section we sharpen the estimate used in [Norros and Reittu 06] for the aggregate capacity of a set of the form $\left\{i: \Lambda_{i}>N^{\gamma}\right\}$. In particular, we obtain that the aggregate capacity of a tier $\mathcal{T}_{\gamma, \gamma+\epsilon(N)}$ is asymptotically overwhelmingly larger than all strictly greater vertex capacities together. This holds also when $\gamma$ depends on $N$.

We use frequently the notation $x+I:=\{x+y: y \in I\}$, where $x \in \mathbb{R}$ and $I \subset \mathbb{R}$ is an interval.

Lemma 3.I. Let $\alpha=\alpha(N) \in(0, \bar{\alpha})$, where $\bar{\alpha}<1 /(\tau-1)$. Then for $N$ sufficiently large,

$$
\mathbb{P}\left(\frac{\sum_{i=1}^{N} \Lambda_{i} 1_{\left\{\Lambda_{i}>N^{\alpha}\right\}}}{N^{1-(\tau-2) \alpha} \mathbb{E}\{\Lambda\}} \in\left(\frac{1}{4}, 4\right)\right) \geq 1-\left(\frac{\mathbb{E}\{\Lambda\}}{2}\right)^{-\tau+1} N^{-(1-(\tau-1) \bar{\alpha})(\tau-2)} .
$$

Proof. Define

$$
p(n)=\mathbb{P}\left(\frac{\frac{1}{n} \sum_{i=1}^{n} \Lambda_{i}}{\mathbb{E}\{\Lambda\}} \notin\left(\frac{1}{2}, 2\right)\right), \quad N_{\alpha}=\sum_{1}^{N} 1_{\left\{\Lambda_{i}>N^{\alpha}\right\}} .
$$


Since $\mathbb{P}[\Lambda>y \mid \Lambda>x]=\mathbb{P}(x \Lambda>y)$, we have the distribution equality

$$
\sum_{i=1}^{N} \Lambda_{i} 1_{\left\{\Lambda_{i}>N^{\alpha}\right\}} \stackrel{\mathcal{D}}{=} N^{\alpha} \sum_{k=1}^{N_{\alpha}} \tilde{\Lambda}_{k}
$$

where the $\tilde{\Lambda}_{k}$ 's are fresh independent copies of $\Lambda$. Then

$$
\begin{aligned}
\mathbb{P}\left(\frac{\frac{1}{N_{\alpha}} \sum_{k=1}^{N_{\alpha}} \tilde{\Lambda}_{k}}{\mathbb{E}\{\Lambda\}} \notin\left[\frac{1}{2}, 2\right]\right) & =\mathbb{E}\left\{p\left(N_{\alpha}\right)\right\} \\
& \leq \mathbb{P}\left(N_{\alpha}<\frac{1}{2} \mathbb{E}\left\{N_{\alpha}\right\}\right)+\mathbb{E}\left\{p\left(N_{\alpha}\right) 1_{\left\{N_{\alpha} \geq \frac{1}{2} \mathbb{E}\left\{N_{\alpha}\right\}\right\}}\right\}
\end{aligned}
$$

The distribution of $N_{\alpha}$ is $\operatorname{Bin}\left(N, N^{-(\tau-1) \alpha}\right)$. The well-known bounds for a binomial random variable $X$,

$$
\begin{aligned}
& \mathbb{P}(X<\mathbb{E}\{X\}-x) \leq \exp \left(-\frac{x^{2}}{2 \mathbb{E}\{X\}}\right) \\
& \mathbb{P}(X>\mathbb{E}\{X\}+x) \leq \exp \left(-\frac{x^{2}}{2 \mathbb{E}\{X\}}+\frac{x^{3}}{\mathbb{E}\{X\}^{3}}\right),
\end{aligned}
$$

yield

$$
\mathbb{P}\left(\frac{N_{\alpha}}{\mathbb{E}\left\{N_{\alpha}\right\}}<\frac{1}{2}\right) \leq e^{-\mathbb{E}\left\{N_{\alpha}\right\} / 8}, \quad \mathbb{P}\left(\frac{N_{\alpha}}{\mathbb{E}\left\{N_{\alpha}\right\}}>2\right) \leq e^{-\mathbb{E}\left\{N_{\alpha}\right\} / 8+1} .
$$

By the well-known result on subexponential variables with finite mean (see, e.g., [Goldie and Klüppelberg 98]),

$$
\mathbb{P}\left(\frac{1}{n} \sum_{i=1}^{n} \Lambda_{i}>2 \mathbb{E}\{\Lambda\}\right) \sim \mathbb{P}\left(\max \left(\Lambda_{1}, \ldots, \Lambda_{n}\right)>n \mathbb{E}\{\Lambda\}\right) \sim \mathbb{E}\{\Lambda\}^{-\tau+1} n^{-\tau+2}
$$

For the large deviation to the opposite direction, the classical Cramér theorem applies, and the probability goes to zero at exponential speed. Thus, for $N$ sufficiently large,

$$
\mathbb{E}\left\{p\left(N_{\alpha}\right)\right\} \leq e^{-\mathbb{E}\left\{N_{\alpha}\right\} / 8}+\frac{3}{2} \mathbb{E}\{\Lambda\}^{-\tau+1}\left(\frac{\mathbb{E}\left\{N_{\alpha}\right\}}{2}\right)^{-\tau+2}
$$

Combining (3.1)-(3.4), replacing $\alpha$ by the worse case $\bar{\alpha}$, and bounding the exponential terms from above by replacing the factor $3 / 2$ in (3.4) by 2 , we get the assertion. 
Lemma 3.2. Let $\alpha_{0}(N), \alpha_{1}(N) \in(0, \bar{\alpha})$, where $\bar{\alpha}<1 /(\tau-1)$ and $\alpha_{0}(N) \leq \alpha_{1}(N)-$ $\epsilon(N)$. Then for $N$ sufficiently large,

$\mathbb{P}\left(\frac{\sum_{i=1}^{N} \Lambda_{i} 1_{\left\{\Lambda_{i} \in\left(N^{\alpha_{0}}, N^{\alpha_{1}}\right]\right\}}}{N^{1-(\tau-2) \alpha_{0}} \mathbb{E}\{\Lambda\}} \in\left(\frac{1}{5}, 5\right)\right) \geq 1-2\left(\frac{\mathbb{E}\{\Lambda\}}{2}\right)^{-\tau+1} N^{-(1-(\tau-1) \bar{\alpha})(\tau-2)}$.

Proof. This follows by applying Lemma 3.1 to both $\alpha_{0}$ and $\alpha_{1}$ and noting that $N^{-(\tau-2)\left(\alpha_{1}-\alpha_{0}\right)} \rightarrow 0$.

Lemma 3.3. For any fixed $b>1$,

$$
\frac{\sum_{i=1}^{N} \Lambda_{i} 1_{\left\{\Lambda_{i}>N^{b \epsilon(N)}\right\}}}{\sum_{i=1}^{N} \Lambda_{i} 1_{\left\{\Lambda_{i}>N^{\epsilon(N)}\right\}}} \rightarrow 0 \quad \text { in probability. }
$$

Proof. By Lemma 3.1,

$$
\frac{\sum_{i=1}^{N} \Lambda_{i} 1_{\left\{\Lambda_{i}>N^{b \epsilon(N)}\right\}}}{\sum_{i=1}^{N} \Lambda_{i} 1_{\left\{\Lambda_{i}>N^{\epsilon(N)}\right\}}} \cdot N^{(b-1)(\tau-2) \epsilon(N)} \in\left[\frac{1}{16}, 16\right] \quad \text { a.a.s. }
$$

and the claim follows, since $N^{\epsilon(N)} \rightarrow \infty$.

Lemma 3.3, combined with our earlier results in [Norros and Reittu 06], entails the important observation that the first contact to the core is a.a.s. close to its bottom:

Proposition 3.4. Fix $b>1$. Let $I_{N}$ be a random node of the giant component of $G_{N}$. Asymptotically almost surely, $I_{N}$ is connected to $\mathcal{I}_{\beta_{k^{*}}, b \beta_{k^{*}}}$ with a path that avoids the set $\left\{i: \Lambda_{i}>N^{b \beta_{k^{*}}}\right\}$, and whose length coincides with the distance between $I_{N}$ and the core.

Proof. Since the proportional size of the core shrinks to zero, it suffices to consider the case that $I_{N}$ is picked from outside the core. By the results in [Norros and Reittu 06], conditional that $I_{N}$ is connected with the core, one of the minimallength paths to the core has its core endpoint distributed as the first core element in an i.i.d. sequence $J_{n} \in\{1, \ldots, N\}$ with common distribution (2.1). Now $\beta_{k^{*}}$ is proportional to $\epsilon(N)$, and Lemma 3.3 can be applied with $\beta_{k^{*}}$ in the role of $\epsilon(N)$. We conclude that the first core element in the sequence $J_{n}$ belongs a.a.s. to the set $\mathcal{T}_{\beta_{k^{*}}, b \beta_{k^{*}}}$, and the statement of the proposition follows. 


\section{Horizontal Paths in the Core}

In this section, we show that quite thin tiers of the form $\mathcal{T}_{\gamma^{\prime}, \gamma^{\prime \prime}}$ are internally (as induced subgraphs) almost connected in the sense that the proportional size of the largest component approaches one, and moreover, the diameter of that largest component is obtained with high accuracy from remarkable results on classical random graphs. For deterministic $\gamma$, the tiers $\mathcal{T}_{\gamma-\epsilon(N), \gamma}$ are a.a.s. connected, and

their diameters can be picked almost unequivocally from the following classical theorem [Bollobás 01].

Theorem 4.I. Consider the Erdös-Rényi random graph $G_{n, p}$ and let $p=p(n)$ and $d=d(n)>2$ satisfy

$$
\begin{gathered}
\frac{\log n}{d}-3 \log \log n \rightarrow \infty, \\
p^{d} n^{d-1}-2 \log n \rightarrow \infty, \\
p^{d-1} n^{d-2}-2 \log n \rightarrow-\infty .
\end{gathered}
$$

Then $G_{n, p}$ has diameter d a.a.s.

To include also lower parts of the core, we apply the following rather recent result of [Chung and Lu 01].

Theorem 4.2. If $n p \rightarrow \infty$ and $(\log n) /(\log n p) \rightarrow \infty$, then almost surely

$$
\operatorname{diam}\left(G_{n, p}\right)=(1+o(1)) \frac{\log n}{\log n p}
$$

where $\operatorname{diam}(G)$ denotes the diameter of the largest (giant) component of $G$.

Define

$$
w(\gamma):=\left\lceil\frac{1-(\tau-1) \gamma}{(3-\tau) \gamma}\right\rceil
$$

Proposition 4.3. Let $\gamma(N)$ be a nonincreasing function such that $\gamma(N) \in\left[\epsilon(N), \frac{1}{2}\right)$ for all $N$. If $\lim \gamma(N)>0$, assume also that $\gamma(\infty)$ does not correspond to a jump of the ceiling function in (4.1). Then there exists another function $\gamma_{*}(N)$ such that

$$
\gamma_{*}(N)<\gamma(N), \quad \gamma_{*}(N) / \gamma(N) \rightarrow 1, \quad\left|\mathcal{T}_{\gamma_{*}(N), \gamma(N)}\right| \rightarrow \infty,
$$

and

$$
\operatorname{diam}\left(\mathcal{T}_{\gamma_{*}, \gamma}\right)=(1+o(1)) w(\gamma(N)) \quad \text { a.a.s. }
$$


where $\operatorname{diam}(\cdot)$ is generally interpreted as in Theorem 4.2. When $\gamma(N)$ is bounded away from zero, $\mathcal{T}_{\gamma_{*}, \gamma}$ is a.a.s. connected and its diameter is a.a.s. equal to $w(\gamma(N))$.

Proof. We shall suppress the $N$ 's in $\gamma(N)$ etc. when it improves the clarity of the presentation.

Considered as an induced subgraph, $\mathcal{T}_{\gamma_{*}, \gamma}$ is denser (respectively sparser) than the classical random graph $G_{n, p_{*}}$ (respectively $\left.G_{n, p^{*}}\right)$ with

$$
n=n(N)=\left|\mathcal{I}_{\gamma_{*}, \gamma}\right|, \quad p_{*}=p_{*}(N)=\frac{N^{2 \gamma_{*}}}{L_{N}}, \quad p^{*}=p^{*}(N)=\frac{N^{2 \gamma}}{L_{N}} .
$$

Set $\gamma_{\delta}(N)=\gamma-\delta \epsilon(N)$, where $\delta \in(0,1)$. We fix $\delta$ for a while and choose $\gamma_{*}=\gamma_{\delta}$. By Lemma $3.2,\left|\mathcal{I}_{\gamma_{*}, \gamma}\right| \rightarrow \infty$, and

$$
\frac{\left|\mathcal{T}_{\gamma_{*}, \gamma}\right|}{N^{1-(\tau-1) \gamma_{*}}} \in\left[\frac{1}{5}, 5\right], \quad \text { a.a.s. }
$$

On the other hand,

$$
\frac{L_{N} / N}{\mathbb{E}\{\Lambda\}} \in\left[\frac{1}{2}, 2\right] \quad \text { a.a.s. }
$$

Thus a.a.s.,

$$
\begin{aligned}
\log n & \in\left(1-(\tau-1) \gamma_{*}\right) \log N+[-\log 4, \log 4], \\
\log \left(n p_{*}\right) & \in(3-\tau) \gamma_{*} \log N-\log \mathbb{E}\{\Lambda\}+[-\log 8, \log 8], \\
\log \left(n p^{*}\right) & \in\left(2 \gamma-(\tau-1) \gamma_{*}\right) \log N-\log \mathbb{E}\{\Lambda\}+[-\log 8, \log 8] .
\end{aligned}
$$

Note that $p^{*}(N) \rightarrow 0$ and $n(N) p_{*}(N) \rightarrow \infty$, so Theorem 4.2 applies to $G_{n, p_{*}}$ and $G_{n, p^{*}}$. We distinguish two cases.

First, if $\gamma(N)$ is bounded away from zero, we are in fact in the regime of Theorem 4.1, and a computation of the diameter $d$ leads to the expression of $w(\gamma)$. Thus, Theorem 4.1 yields the inequalities

$$
\begin{aligned}
& \operatorname{diam}\left(\mathcal{T}_{\gamma_{*}, \gamma}\right) \leq\left\lceil\frac{1-(\tau-1) \gamma_{*}}{(3-\tau) \gamma_{*}}\right\rceil \quad \text { a.a.s., } \\
& \operatorname{diam}\left(\mathcal{T}_{\gamma_{*}, \gamma}\right) \geq\left\lceil\frac{1-(\tau-1) \gamma_{*}}{2 \gamma+(1-\tau) \gamma_{*}}\right\rceil \quad \text { a.a.s. }
\end{aligned}
$$

Since the right-hand sides are with sufficiently large $N$ both equal to the righthand side of $w(\gamma)$, the claim follows. The value of $\delta$ did not matter.

The second case is that $\gamma(N) \searrow 0$. Since $n(N) p_{*}(N) \rightarrow \infty$, the subgraph still has a giant component whose proportional size approaches one, although it is 
not necessarily connected. Choose any sequence $\delta_{k} \searrow 0$, and set

$$
\eta_{k}=\max \left\{\frac{(3-\tau) \gamma}{(3-\tau) \gamma_{\delta_{k}}}, \frac{2 \gamma+(\tau-1) \gamma_{\delta_{k}}}{(3-\tau) \gamma}\right\} .
$$

Note that $\eta_{k} \rightarrow 1$. Now define a sequence $N_{k}$ as

$$
\begin{aligned}
N_{k}=\inf \{ & M: \mathbb{P}\left(\left|\mathcal{T}_{\gamma_{\delta_{k}}, \gamma}\right| \frac{N^{2 \gamma_{\delta_{k}}}}{L_{N}} \geq k\right) \geq 1-\frac{1}{k} \quad \forall N \geq M, \\
& \left.\mathbb{P}\left(\operatorname{diam}\left(\mathcal{T}_{\gamma_{\delta_{k}}, \gamma}\right) \in\left(\eta_{k}^{-2}, \eta_{k}^{2}\right) \cdot \frac{1}{(3-\tau) \gamma}\right) \geq 1-\frac{1}{k} \quad \forall N \geq M\right\} .
\end{aligned}
$$

Thanks to Theorem 4.2 , the $N_{k}$ 's are finite numbers, and obviously they grow without bound. Finally, set

$$
k(N):=\max \left\{k: N_{k} \leq N\right\}
$$

and choose $\gamma_{*}(N)=\gamma_{\delta_{k(N)}}$. Then obviously

$$
\operatorname{diam}\left(\mathcal{T}_{\gamma_{*}, \gamma}\right)=(1+o(1)) \frac{1}{(3-\tau) \gamma(N)} \quad \text { a.a.s. }
$$

completing the proof.

In a similar way, we can prove a diameter result for the tiers $V_{k}=V_{k}^{(N)}$, defined at the end of Section 2:

Proposition 4.4. The tiers $V_{0}, \ldots, V_{k^{*}}$, considered as induced subgraphs of $G_{N}$, are almost connected in the sense that the relative sizes of their largest components approach 1 as $N \rightarrow \infty$, and their diameters satisfy, a.a.s.,

$$
\begin{aligned}
& \operatorname{diam}\left(V_{0}\right)=0, \\
& \operatorname{diam}\left(V_{1}\right)=2, \\
& \operatorname{diam}\left(V_{k}\right) \in(1+o(1))\left[w\left(\beta_{k-1}\right), w\left(\beta_{k}\right)\right], \quad k=2, \ldots, k^{*} .
\end{aligned}
$$

By Proposition 4.3, we can say that the width of the core at height $N^{\gamma}$, measured by hop distance, is $w(\gamma)$. Moreover, this holds also for varying $\gamma=$ $\gamma(N)$ down to the bottom of the core.

When $\gamma \in\left(0, \frac{1}{2}\right)$, we have

$$
w((\tau-2) \gamma)>w(\gamma)+2
$$

This has the following heuristic consequence. For connecting two vertices with capacities $\approx N^{\gamma}$, a "horizontal" path staying at about the same height in the 
core is longer than a path that jumps at both ends with one hop to the height $N^{(\tau-2) \gamma}$ and finds the horizontal connection at that level. On the other hand, this is how high in the core the neighborhood of a vertex typically reaches. Thus, a horizontal move should be made at as high a level as possible.

\section{The Main Result}

We have now essentially collected all elements needed to understand the situation in which a top part of the core is deleted. First, since the aggregate capacity of any top part is concentrated at its bottom, the remains of the core are found from outside almost as easily as the original core was found. Second, if a lower part of the core is alive, we can find "vertical" paths from the bottom of the core to a top tier of its remaining part, as we did with the undamaged core in [Norros and Reittu 06] (a complete proof of this step requires a slight modification of the proof of [Norros and Reittu 06, Proposition 4.3] and is presented below in detail). Third, Proposition 4.3 guarantees the existence of a "horizontal" path between almost any pair of vertices within this top tier, and provides also an explicit upper bound for its length. It remains to couple these pieces together.

Theorem 5.I. Let $\gamma:=\gamma(N) \in\left(\epsilon(N), \frac{1}{2}\right)$ be such that $\gamma(N)$ is nonincreasing and $\gamma(N) / \epsilon(N)$ nondecreasing with respect to $N$. Denote by $H_{\gamma}=H_{\gamma}^{(N)}$ the graph obtained from $G_{N}$ by deleting all vertices with capacity greater than $N^{\gamma}$, together with the edges attached to them. The following hold:

1. The graph $H_{\gamma}$ has a.a.s. a giant component whose relative size approaches the relative size of the giant component of $G_{N}$.

2. The distance between two randomly chosen vertices of the giant component of $H_{\gamma}$ is a.a.s. less than

$$
(1+o(1))\left(\frac{2}{-\log (\tau-2)}\left(\log \log N-\log \frac{1}{\gamma}\right)+w(\gamma)\right),
$$

where $w(\gamma)$ is given in (4.1).

Proof. (1) Define

$$
\begin{aligned}
\gamma_{0} & =\gamma, \\
\gamma_{1} & =\gamma_{0}-\epsilon(N), \\
\gamma_{k+1} & =(\tau-2) \gamma_{k}+\epsilon(N), \quad k=1,2, \ldots,
\end{aligned}
$$


and let

$$
\bar{k}=\min \left\{k \geq 1: \gamma_{k} \leq \frac{4-\tau}{3-\tau} \epsilon(N)\right\}
$$

It is easy to check (see (4) below) that $\bar{k}=O(\log \log N)$.

Consider the tiers $\mathcal{T}_{\gamma_{1}, \gamma_{0}}, \mathcal{T}_{\gamma_{2}, \gamma_{1}}, \ldots, \mathcal{T}_{\gamma_{\bar{k}}, \gamma_{\bar{k}-1}}$. The idea of the proof is to apply the diameter result, Theorem 4.2 , to $\mathcal{T}_{\gamma_{1}, \gamma_{0}}$, and if $\bar{k} \geq 2$, to imitate our proof of Theorem 2.3 to show that vertices of tier $\mathcal{T}_{\gamma_{\bar{k}}, \gamma_{\bar{k}-1}}$ are with high probability connected to $\mathcal{T}_{\gamma_{1}, \gamma_{0}}$ with a path jumping from tier to tier.

(2) Assume that $\bar{k} \geq 2$. Let $I_{0}=I_{0}(N)$ be a stochastic (not necessarily uniformly random) vertex of $\mathcal{T}_{\gamma_{\bar{k}}, \gamma_{\bar{k}-1}}$ such that conditioned on $\boldsymbol{\Lambda}, I_{0}$ is independent of the edges within $\bigcup_{1}^{\bar{k}} \mathcal{T}_{\gamma_{j}, \gamma_{j-1}}$. Define the sequence of vertices $I_{1}, I_{2}, \ldots$ recursively as follows. If $I_{n} \in \mathcal{T}_{\gamma_{\bar{k}-n}, \gamma_{\bar{k}-n-1}}$, then $I_{n+1}$ is a neighbor (say with smallest index) of $I_{n}$ belonging to $\mathcal{T}_{\gamma_{\bar{k}-n-1}, \gamma_{\bar{k}-n-2}}$ if such a neighbor exists. Otherwise, $I_{n+1}=I_{n}$, and the rest of the sequence repeats $I_{n}$ as well. Define

$$
\begin{aligned}
A_{n}= & \left\{I_{n} \in \mathcal{T}_{\gamma_{\bar{k}-n}, \gamma_{\bar{k}-n-1}}\right\}, \quad n=0,1, \ldots, \bar{k}-1, \\
B^{\prime}= & \left\{\frac{L_{N}}{N} \leq 2 \mathbb{E}\{\Lambda\}\right\} \\
B_{k}= & \left\{L\left(\mathcal{T}_{\gamma_{k+1}, \gamma_{k}}\right) \in \mathbb{E}\{\Lambda\} N^{1-(\tau-2) \gamma_{k}}\left(\frac{1}{5}, 5\right)\right\}, \quad k=0, \ldots, \bar{k}-1, \\
& \text { where } L(S):=\sum_{i \in S} \Lambda_{i}, \\
B= & B^{\prime} \cap B_{0} \cap \cdots \cap B_{\bar{k}-1} .
\end{aligned}
$$

We have $A_{\bar{k}-1} \subseteq A_{\bar{k}-2} \subseteq \cdots \subseteq A_{0}$. Proposition 3.2 yields

$$
\mathbb{P}\left(B_{0} \cap \cdots \cap B_{\bar{k}-1}\right) \geq 1-2 \bar{k}\left(\frac{\mathbb{E}\{\Lambda\}}{2}\right)^{-\tau+1} N^{-(1-(\tau-1) \gamma)(\tau-2)} \rightarrow 1
$$

as $N \rightarrow \infty$. On $B$ in turn, we have for each $n$ by Proposition 3.2 that

$$
\begin{aligned}
\mathbb{P}\left[A_{n+1}^{c} \mid \boldsymbol{\Lambda}, A_{n}\right] & =\mathbb{E}\left[\exp \left(-\frac{\Lambda_{I_{n}} L\left(\mathcal{T}_{\gamma_{\bar{k}-n-1}, \gamma_{\bar{k}-n-2}}\right)}{L_{N}}\right) \mid \boldsymbol{\Lambda}, A_{n}\right] \\
& \leq \exp \left(-\frac{1}{2 \mathbb{E}\{\Lambda\} N} N^{\gamma_{\bar{k}-n}} \cdot \frac{1}{5} N^{1-(\tau-2) \gamma_{\bar{k}-n-1}}\right) \\
& \leq \exp \left(-\frac{e^{\ell(N)}}{10 \mathbb{E}\{\Lambda\}}\right)
\end{aligned}
$$


It follows that on $B$,

$$
\begin{aligned}
\mathbb{P}\left[A_{\bar{k}-1} \mid \boldsymbol{\Lambda}, A_{0}\right] & =\prod_{n=0}^{\bar{k}-1} \mathbb{P}\left[A_{n+1}^{c} \mid \boldsymbol{\Lambda}, A_{n}\right] \\
& \geq\left(1-\exp \left(-\frac{e^{\ell(N)}}{10 \mathbb{E}\{\Lambda\}}\right)\right)^{\bar{k}} \\
& \geq 1-\bar{k} \exp \left(-c e^{\ell(N)}\right) \\
& =1-\frac{\bar{k}}{\log ^{2} N} \exp \left(\log ^{3} N-c\left(e^{\log ^{4} N}\right)^{\ell(N) / \log ^{4} N}\right) \\
& =1-\frac{\bar{k}}{\log ^{2} N} \exp \left(-\log ^{3} N\left(c\left(\log ^{3} N\right)^{\ell(N) / \log ^{4} N-1}-1\right)\right) \\
& \rightarrow 1 \quad \operatorname{as} N \rightarrow \infty
\end{aligned}
$$

by the assumption that $\ell(N) / \log ^{4} N \rightarrow \infty$.

(3) Now let $I$ and $I^{\prime}$ be two independent uniformly randomly chosen vertices of $G_{N}$. By Proposition 3.4, $I$ is a.a.s. either outside the largest component of $G_{N}$ or connected to $\mathcal{T}_{\gamma_{\bar{k}}, \gamma_{\bar{k}-1}}$ with a path that avoids the set $\left\{i \leq N: \Lambda_{i}>N^{\gamma_{\bar{k}-1}}\right\}$ and whose length is at most $\kappa(N)$. Denote the endpoint of that path by $I_{0}$. In the case that $\bar{k} \geq 2$, step (2) above showed that $I_{0}$ is a.a.s. connected to a vertex $I_{\bar{k}-1} \in \mathcal{T}_{\gamma_{1}, \gamma_{0}}$ over a path of length $\bar{k}$. The same claim holds for $I^{\prime}$, with vertices $I_{0}^{\prime}$ and $I_{\bar{k}-1}^{\prime}$ respectively. Now, $I_{\bar{k}-1}$ and $I_{\bar{k}-1}^{\prime}$ belong a.a.s. to the largest component of the induced subgraph $\mathcal{T}_{\gamma_{1}, \gamma_{0}}$, and Theorem 4.2 yields that the distance between them is at most $(1+o(1)) w(\gamma)$.

(4) We have now shown that if $I$ and $I^{\prime}$ both belong to the largest component of $G_{N}$, then the distance between them is a.a.s. at most

$$
(1+o(1))(2 \bar{k}+w(\gamma))
$$

It remains to show that $\bar{k}$ can be replaced in this expression by

$$
\frac{\log \log N-\log (1 / \gamma)}{-\log (\tau-2)}
$$

First, if $\gamma(N) \leq C \epsilon(N)$ for all $N$ and some finite $C>1$, then

$$
\log \frac{1}{\gamma} \in(\log \log N-\log \ell(N))+[-\log C, 0],
$$

and (5.2) is in this region negligible compared with $w(\gamma)$. On the other hand, $\bar{k}$ is in this region limited by a constant, whereas $w(\gamma(N)) \rightarrow \infty$. Thus, the assertion of the theorem holds. 
Assume then that $\gamma(N) / \epsilon(N)$ is unbounded. Then $\gamma_{1}>((4-\tau) /(3-\tau)) \epsilon(N)$ for large $N$, so that $\bar{k} \geq 2$. Since

$$
\gamma_{k+1}=(\tau-2)^{k} \gamma_{1}+\epsilon(N) \sum_{i=0}^{k-1}(\tau-2)^{i},
$$

a short computation (using the geometric series formula) yields that the condition $\gamma_{k} \leq((4-\tau) /(3-\tau)) \epsilon(N)$ is equivalent to the condition

$$
(\tau-2)^{k}\left(\gamma_{1}-\frac{\epsilon(N)}{3-\tau}\right) \leq \epsilon(N),
$$

and further to

$$
\begin{aligned}
k & \geq \frac{1}{-\log (\tau-2)}\left(\log \log N+\log \left(\gamma(N)-\frac{4-\tau}{3-\tau} \epsilon(N)\right)-\log \ell(N)\right) \\
& =(1+o(1)) \frac{\log \log N-\log (1 / \gamma(N))}{-\log (\tau-2)} .
\end{aligned}
$$

Thus, $\bar{k}$ equals the last, asymptotic, expression. This finishes the proof.

Theorem 5.1 tells that removing a top part of the core down to $N^{\gamma}$ with fixed $\gamma>0$ has no effect on the asymptotic upper bound (5.1). On the other hand, with $\gamma(N) \propto \epsilon(N)$ we obtain

$$
w(\gamma(N)) \propto \frac{1}{(3-\tau) \epsilon(N)}=\frac{\log N}{(3-\tau) \ell(N)} .
$$

Combining this with Proposition 3.4, we find that if the whole core is removed, the remaining graph still has a giant component with the same proportional size as the original and diameter proportional to $\log (N) / \ell(N)$, slightly smaller than that of a (supercritical) power-law graph with $\tau>3$. Asymptotics of intermediate cases between these two extremes can be computed from (3.4) as well.

Acknowledgments. This work was supported by the Tekes projects ABI and IPLU-II.

\section{References}

[Albert et al. 00] R. Albert, H. Jeong, and A.-L. Barabási. "Error and Attack Tolerance of Complex Networks." Nature 406 (2000), 378-381.

[Bollobás 01] B. Bollobás. Random Graphs, 2nd ed. Cambridge, UK: Cambridge University Press, 2001. 
[Bollobás and Riordan 03] B. Bollobás and O. Riordan. "Robustness and Vulnerability of Scale-Free Random Graphs." Internet Mathematics 1:1 (2003), 1-35.

[Chung and Lu 01] F. Chung and L. Lu. "The Diameter of Sparse Random Graphs." Adv. Appl. Math. 26 (2001), 257-279.

[Chung and Lu 03] F. Chung and L. Lu. "The Average Distance in a Random Graph with Given Expected Degrees." Internet Mathematics 1 (2003), 91-114.

[Goldie and Klüppelberg 98] C. M. Goldie and C. Klüppelberg. "Subexponential Distributions." A Practical Guide to Heavy Tails, editied by R. J. Adler, R. E. Feldman, and M. S. Taqqu, pp. 435-460. Boston: Birkhäuser, 1998.

[van der Hofstad and Hooghiemstra 07] R. van der Hofstad and G. Hooghiemstra. "Diameters in Preferential Attachment Models." Paper presented at the Fourteenth Applied Probability Society of INFORMS Conference, Eindhoven, The Netherlands, July 9-11, 2007. Available at http://ssor.twi.tudelft.nl/ gerardh.

[Newman et al. 01] M. E. J. Newman, S. H. Strogatz, and D. J. Watts. "Random Graphs with Arbitary Degree Distribution and Their Applications." Phys. Rev. E 64 (2001), 026118.

[Norros and Reittu 06] I. Norros and H. Reittu. "On a Conditionally Poissonian Graph Process." Adv. Appl. Prob. 38 (2006), 59-75.

[Reittu and Norros 04] H. Reittu and I. Norros. "On the Power-Law Random Graph Model of Massive Data Networks." Performance Evaluation 55 (2004), 3-23.

Ilkka Norros, VTT Technical Research Centre of Finland, P.O. Box 1000, 02044 VTT, Finland (ilkka.norros@vtt.fi)

Hannu Reittu, VTT Technical Research Centre of Finland, P.O. Box 1000, 02044 VTT, Finland (hannu.reittu@vtt.fi)

Received January 10, 2008; accepted March 3, 2009. 